\title{
Structural sensitivity analysis in nonlinear and transient problems using the local response function technique
}

\author{
Marcin Marek Kamiński
}

Received: 11 October 2009 / Revised: 10 August 2010 / Accepted: 11 August 2010 / Published online: 1 September 2010

(C) The Author(s) 2010. This article is published with open access at Springerlink.com

\begin{abstract}
The main aim of this work is the mathematical formulation, computational implementation and the application of the local version of the Response Function Method (RFM) to analyze structural design sensitivity in nonlinear structures and problems. This method is based on the Finite Element Method-based determination of the polynomial response function between design parameter and the structural state function like displacements or temperatures. One may use this numerical technique in its global version, where a single polynomial is determined for the entire computational domain or, in the case of nonlinear, transient analyses or the heterogeneous domains, in the local approach - where nodal response function are to be determined. The application of this methodology is illustrated with three examples - transient heat transfer in the homogeneous rod, the elastoplastic analysis of 2D truss as well as the eigenvibrations for a large scale 3D structure, where time, increment and eigenvalue dependent variations of the first and the second order sensitivities with respect to the physical and material parameters are computed. The first order gradients computed with the use of the RFM approach are contrasted with the finite difference computations.
\end{abstract}

Keywords Sensitivity analysis - Response Function Method · Transient heat transfer - Elastoplastic analysis . Eigenvalue analysis $\cdot$ Finite Element Method

\footnotetext{
M. M. Kamiński (凶)

Department of Steel Structures, Faculty of Civil Engineering, Architecture and Environmental Engineering, Technical University of Łódź, Al. Politechniki 6, 90-924 Łódź, Poland e-mail: Marcin.Kaminski@p.lodz.pl
}

\section{Introduction}

Verification of the structural design sensitivity of nonlinear systems is a very important and interesting issue in modern computational mechanics since the unsteady boundary conditions and inelastic or time-dependent responses of the real structures observed most frequently in engineering practice. Furthermore, the linear systems sensitivity coefficients, and also their general character can be predicted using the engineering intuition, while the sensitivity of nonlinear systems is much more complex; the general interest in this area is expressed also by many recent works referenced in Kowalczyk (2006). Contrary to the existing structural sensitivity analysis methods, like the Adjoint Variable Method (AVM), Direct Differentiation Method (DDM) or Central Finite Difference Method (CFD) or even automatic differentiation algorithms for the entire computer systems, the Response Function Method (RFM), quite similar to the Response Surface Methods (RSM) (Khuri and Cornell 1987), has been developed for an accurate and essentially easier determination of higher order sensitivities as well as considering its application with various software with no opportunity to access the source code itself; the last issue is of a special importance for the Finite Element Method academic users, at least. Those higher order sensitivities are of the paramount interest in the stochastic perturbation technique, to determine precisely higher probabilistic moments and to assure very accurate probabilistic convergence for the expectations and standard deviations. It remains clear that this method may successfully enable joint determination of the sensitivity gradients and probabilistic moments for the given design parameters/input random variables (Kamiński 
2009b). The general idea of this method essentially differs from all of the previous approaches, where the final sensitivity gradients of structural response were determined in a discrete sense and were dependent on design parameter increment (CFDM), on all discrete partial derivatives of the system matrices with respect to the design parameter (although analytically determined in DDM) and on the solution of some extra equilibrium equations systems (like in the AVM). Now the key issue is to determine the nodal analytical functions relating the structural response in the given point with the specified input parameter also by the Finite Element Method. It is done using the standard approximation technique from several solutions of the original problem around the mean value of the design parameter. The polynomial nodal responses are the basis for further, also analytical, determination of first and higher order sensitivities to this design parameter. Application of this method in the global formulation was possible in linear elastostatics (Kamiński 2009a), where the polynomial form of the structural response was relatively easy predictable but analysis of transient (Kang et al. 2006), nonlinear problems (Kleiber et al. 1997) as well as heterogeneous structures or those with local time-dependent parameters needs necessarily the local (even time dependent) approach. The application of the local version is demonstrated here for transient heat transfer in homogeneous rod, the in-plane elastoplastic deformation of relatively simple 2D truss and the eigenvibrations analysis for the large scale linear elastic 3D telecommunication structure. The time dependent (in the first case), the increment dependent (in the second example) as well as the eigenvalue number-dependent (in the third case) response functions are determined to compare the first and the second order partial derivatives with respect to such design parameters like heat conductivity, heat capacity and Young modulus. Let us note that computational implementation has hybrid character-the academic and commercial FEM code was used to perform the standard tests, while symbolic computer algebra system MAPLE, v. 11, was employed to make the postprocessing procedures - polynomial approximation, analytical double differentiation and graphical representation of the results (Kamiński 2009a). A verification of the proposed method effectiveness is completed here by a comparison against the CFD scheme. It returns for all analysis types and FEM examples almost perfect agreement of both techniques. As it is documented by the previous applications (see Kamiński 2009b), the Response Function Method may be successfully applied to sensitivity analysis of strongly heterogeneous media like periodic fiber-reinforced composites.

\section{Governing equations for the sensitivity analysis}

2.1 Transient heat transfer and its sensitivity coefficients

Generally, transient heat flow problem consists in determining the temperature field $T=T(\mathbf{x}, \tau)$ governed by the following differential equation:

$\rho c \dot{T}-\left(\lambda_{i j} T,{ }_{j}\right)_{, i}-g=0 ; \mathrm{x}_{\mathrm{i}} \in \Omega ; \tau \in[0, \infty)$,

where $c$ is the heat capacity of the region $\Omega, \rho$ is the density of the material contained in $\Omega, \lambda_{i j}$ is the thermal conductivity second order tensor, while $g$ is the rate of heat generated per unit volume; the variables $T$ and $\tau$ denote temperature field values and time, respectively. This equation should fulfil the boundary conditions of the $\partial \Omega$, which are given as follows:

1. temperature (essential) boundary conditions

$$
\mathrm{T}=\hat{\mathrm{T}} ; \mathrm{x} \in \partial \Omega_{\mathrm{T}},
$$

and for $\partial \Omega_{q}$ part of the total $\partial \Omega$ :

2. heat flux (natural) boundary conditions

$$
\frac{\partial \mathrm{T}}{\partial \mathrm{n}_{\mathrm{i}}}=\hat{\mathrm{q}}_{\mathrm{i}} ; \quad \mathrm{x} \in \partial \Omega_{\mathrm{q}}
$$

where $\partial \Omega_{\mathrm{T}} \cup \partial \Omega_{\mathrm{q}}=\partial \Omega$ and $\partial \Omega_{\mathrm{T}} \cap \partial \Omega_{\mathrm{q}}=\{\varnothing\}$.

The initial conditions are proposed here as

$\mathrm{T}^{0}=\mathrm{T}\left(\mathrm{x}_{\mathrm{i}} ; 0\right) ; \mathrm{x}_{\mathrm{i}} \in \Omega, \tau=0$.

Let us consider further some continuous temperature variations $\delta \mathrm{T}\left(\mathrm{x}_{\mathrm{i}}\right)$ defined in the interior of the region $\Omega$ and vanishing on $\partial \Omega_{\mathrm{T}}$. The alternative, variational formulation of the problem given by (1-4) may be proposed as

$$
\begin{aligned}
& \int_{\Omega}\left(\rho c \dot{T} \delta T+\lambda_{i j} T_{, j} \delta T_{, i}-g \delta T\right) d \Omega-\int_{\partial \Omega_{q}} \hat{q} \delta T d(\partial \Omega) \\
& \quad=0 ; \quad \mathrm{x}_{\mathrm{i}} \in \Omega ; \quad \tau \in[0, \infty) .
\end{aligned}
$$

The equation stated below is the transient formulation of the principle of virtual temperatures and it will be the basis to obtain the temperature sensitivity gradients with respect to some design parameter of the system denoted by $h$. Its straightforward partial differentiation with respect to $h$ 
gives (this differentiation is sometimes noted in right upper index after a comma)

$$
\begin{aligned}
\int_{\Omega} & \left(\rho^{0} c^{0} \dot{T}^{, h} \delta T+\lambda_{i}^{0} T_{, j}^{, h} \delta T_{, i}\right) d \Omega \\
= & \int_{\partial \Omega_{q}} \hat{q}^{, h} \delta T d(\partial \Omega)+\int_{\Omega} g^{, h} \delta T d \Omega \\
& -\int_{\Omega}\left(\left(\rho^{, h} c^{0}+\rho^{0} c^{, h}\right) T^{0} \delta T+\lambda_{i j}^{, h} T_{, j}^{0} \delta T_{, i}\right) d \Omega,
\end{aligned}
$$

The left hand side components at (6) usually differ from 0 , when the right hand side strongly depend on the choice of the design parameter; when a group of the design parameters is considered, this differentiation must proceed with respect to all the design parameters vector components of course. Since this equation corresponds to the transient problem, the gradients determined should also be time dependent.

\subsection{Sensitivity gradients in nonlinear elasticity}

The following boundary value problem is considered:

$$
\begin{aligned}
& \Delta \sigma_{k l, l}+\rho \Delta f_{k}=0 ; \mathbf{x} \in \Omega \\
& \begin{aligned}
\Delta \tilde{\sigma}_{k l}= & C_{k l m n} \Delta \varepsilon_{m n} ; \mathbf{x} \in \Omega \\
\Delta \varepsilon_{m n}=\frac{1}{2}[ & \Delta u_{k, l}+\Delta u_{l, k}+u_{i, k} \Delta u_{i, l}+\Delta u_{i, k} u_{i, l} \\
& \left.\quad+\Delta u_{i, k} \Delta u_{i, l}\right] ; \mathbf{x} \in \Omega
\end{aligned}
\end{aligned}
$$

with the boundary conditions

$$
\begin{aligned}
& \Delta \sigma_{\bar{k} l} n_{l}=\Delta t_{\bar{k}} ; \mathbf{x} \in \partial \Omega_{\sigma}, \bar{k}=1,2,3 \\
& \Delta u_{\hat{k}}=\Delta \hat{u}_{\hat{k}} ; \mathbf{x} \in \partial \Omega_{u}, \hat{k}=1,2,3
\end{aligned}
$$

This problem is solved for the displacement vector $u_{k}(\mathbf{x})$, the strain tensor $\varepsilon_{k l}(\mathbf{x})$ and the stress tensor $\sigma_{k l}(\mathbf{x})$ fulfilling the system (11-13). Let us note that the stress tensor increments $\Delta \sigma_{k l}(\mathbf{x}), \Delta \tilde{\sigma}_{k l}(\mathbf{x})$ denote here the first and the second Piola-Kirchhoff tensors (Kleiber 1985)

$$
\Delta \sigma_{k l}=\Delta F_{k m} \Delta \tilde{\sigma}_{m l}+F_{k m} \Delta \tilde{\sigma}_{m l}+\Delta F_{k m} \tilde{\sigma}_{m l} ; \mathbf{x} \in \Omega
$$

where

$$
\Delta F_{k m}=\Delta u_{k, m} ; \mathbf{x} \in \Omega
$$

The following functional defined on $\Delta u_{k}$ is introduced in order to obtain a numerical solution to this problem:

$$
\begin{aligned}
& J\left(\Delta u_{k}\right) \\
& =\int_{\Omega}\left(\frac{1}{2} C_{k l m n} \Delta \varepsilon_{k l} \Delta \varepsilon_{m n}+\frac{1}{2} \tilde{\sigma}_{k l} \Delta u_{i, k} \Delta u_{i, l}\right. \\
& \left.\quad-\rho \Delta f_{k} \Delta u_{k}\right) d \Omega-\int_{\partial \Omega} \Delta \hat{t}_{k} \Delta u_{k} d(\partial \Omega)
\end{aligned}
$$

As far as the material parameters of the system may be considered as the design parameters, one may write the additional partial derivatives of the energy functional as

$$
\begin{gathered}
\frac{\partial J\left(\Delta u_{k}\right)}{\partial h} \\
=\int_{\Omega}\left(\frac{1}{2} \frac{\partial C_{k l m n}}{\partial h} \Delta \varepsilon_{k l} \Delta \varepsilon_{m n}+\frac{1}{2} \frac{\partial \tilde{\sigma}_{k l}}{\partial h} \Delta u_{i, k} \Delta u_{i, l}\right. \\
\left.\quad-\frac{\partial \rho}{\partial h} \Delta f_{k} \Delta u_{k}\right) d \Omega-\int_{\partial \Omega} \frac{\partial \Delta \hat{t}_{k}}{\partial h} \Delta u_{k} d(\partial \Omega) \\
+\int_{\Omega}\left(\frac{1}{2} C_{k l m n} \frac{\partial \Delta \varepsilon_{k l}}{\partial h} \Delta \varepsilon_{m n}+\frac{1}{2} \tilde{\sigma}_{k l} \frac{\partial \Delta u_{i, k}}{\partial h} \Delta u_{i, l}\right. \\
+\int_{\Omega}\left(\frac{1}{2} C_{k l m n} \Delta \varepsilon_{k l} \frac{\partial \Delta \varepsilon_{m n}}{\partial h}+\frac{1}{2} \tilde{\sigma}_{k l} \Delta u_{i, k} \frac{\partial \Delta u_{i, l}}{\partial h}\right. \\
\left.\quad-\rho \Delta f_{k} \frac{\partial \Delta u_{k}}{\partial h}\right) d \Omega
\end{gathered}
$$

As it is known, the solution to (7--11) proceeds as the minimization of the functional $J\left(\Delta u_{k}\right)$, i.e.

$\delta J\left(\Delta u_{k}\right)=\frac{\partial J}{\partial \Delta u_{k}} \delta\left(\Delta u_{k}\right)=0$.

Let us note that further methodology is valid for both homogeneous as well as heterogeneous materials. The heterogeneous materials case (like composites or the functionally graded materials) allows for the additional integration procedures performed separately over the homogeneous constituents, their external boundaries as well as over the interface surfaces between the components remaining in the contact. 


\section{Computational implementation}

\subsection{Transient heat transfer discretization}

Let us assume that the region $\Omega$ is discretized by the use of the set of finite elements and that the scalar temperature field $T$ is described by the nodal temperatures vector $\theta_{\alpha}$ (Bathe 1996; Oden 1972)

$T\left(x_{i}\right)=H_{\alpha}\left(x_{i}\right) \quad \theta_{\alpha} ; \mathrm{i}=1,2 ; \alpha=1,2, \ldots, \mathrm{N}$,

where $\mathrm{N}$ is the total number of degrees of freedom introduced. The temperature derivatives can be written in the form

$T_{, i}=H_{\alpha, i} \theta_{\alpha}, \mathrm{i}=1,2$.

Moreover, let us introduce the heat capacity matrix $C_{\alpha \beta}$, the heat conductivity matrix $K_{\alpha \beta}$ and the vector $P_{\alpha}$ as follows:

$$
\begin{aligned}
& C_{\alpha \beta}=\int_{\Omega} \rho c H_{\alpha} H_{\beta} d \Omega, \\
& K_{\alpha \beta}=\int_{\Omega} \lambda_{i j} H_{\alpha, i} H_{\beta, j} d \Omega
\end{aligned}
$$

and

$$
P_{\alpha}=\int_{\Omega} g H_{\alpha} d \Omega+\int_{\partial \Omega} \hat{q} H_{\alpha} d \Omega
$$

Next, let us introduce these matrixes into the variational formulation (5) to obtain the following algebraic equations system:

$$
C_{\alpha \beta} \dot{\theta}_{\beta}+K_{\alpha \beta} \theta_{\beta}=P_{\alpha}
$$

The main issue in transient problems is the additional time discretization using some time increment $\Delta \mathrm{t}$. Then we can rewrite the last equation in the following manner:

$$
C_{\alpha \beta} \frac{\theta_{\beta}(t+\Delta t)-\theta_{\beta}(t)}{\Delta t}+K_{\alpha \beta} \theta_{\beta}(t)=P_{\alpha} .
$$

Although we use the explicit method, where the nodal temperatures vector in the second component is taken at the beginning of this time step. However, it is possible to introduce the extra coefficient $0 \leq \delta \leq 1$ to include in this term the temperatures vector after the time step also. There holds

$$
\begin{aligned}
& C_{\alpha \beta} \frac{\theta_{\beta}(t+\Delta t)-\theta_{\beta}(t)}{\Delta t} \\
& \quad+K_{\alpha \beta}\left\{\delta \theta_{\beta}(t+\Delta t)+(1-\delta) \theta_{\beta}(t)\right\}=P_{\alpha},
\end{aligned}
$$

where $\delta=0$ is equivalent to the explicit method, $\delta=1 / 2$ serves for the Crank-Nicholson method, $\delta=2 / 3$ stands for the Galerkin method and at last $\delta=1$ is used in the implicit method (one can use this algorithm with $\delta$ as the extra input parameter).

Let us focus first on the Direct Differentiation Method approach to the sensitivity analysis in the framework of the Finite Element Method implementation. After solution of the basic equilibrium equations of the transient heat flow problem:

$C_{\alpha \beta}^{0} \dot{\theta}_{\beta}^{0}+K_{\alpha \beta}^{0} \theta_{\beta}^{0}=P_{\alpha}^{0}$,

we need to solve for the temperature sensitivities using the modification of (24) obtained by the partial differentiation; there holds

$C_{\alpha \beta} \dot{\theta}_{\beta}^{, h}+K_{\alpha \beta} \theta_{\beta}^{, h}=P_{\alpha}^{, h}-\left(C_{\alpha \beta}^{, h} \dot{\theta}_{\beta}+K_{\alpha \beta}^{, h} \theta_{\beta}\right)$,

The differentiation of the system matrices depend on the choice of the design parameter and is done analytically together with the formation of heat capacity and heat conductivity matrices. Since not always there is a direct access to those matrices, the new technique is proposed, which does not need any access to the source code of the FEM system. It is based on the primary approximation of the analytical interrelation between some nodal temperature and the design parameter considered. This polynomial approximation is obtained from the several traditional solutions of the initial transient problem solved around the mean value of the parameter $h$, additional computation of this polynomial coefficients and final numerical determination of the first (and also higher) order derivative for the approximated functions. Undoubtedly, the RFM computational strategy takes more computational time than the CFD, for instance, since the few (usually not less than 10) solutions of the original problem are necessary (contrary to 2 needed in the CFD). The post-processing procedures cost remains almost the same in the view of symbolic computer algebra employment. However, the difference between the computational time cost starts to reduce when higher order sensitivities (partial derivatives) are to be computed because the RFM preserves the initial time amount, while the CFD cost increases together with the required order derivative. Furthermore, the RFM recovers all the sensitivities with the same errors reflecting the approximation method, whereas the numerical error in the CFD also increases with an order increase. Quite separate computational studies are necessary to contrast various approximation methods in RFM against the CFD or analytical determination of the particular sensitivity coefficients to detect the most efficient approaches.

The FEM formulation for the method called the Response Function Method (RFM) is to recover the nth 
order polynomial approximation of the temperature in a given node with respect to the input random variable $b$ of the mesh in the following form (Kamiński 2009a, b):

$\theta_{\beta}=D_{\beta m} b^{m}, \quad m=0, \ldots, n-1 ; \beta=1, \ldots, N$.

so that there holds

$$
\begin{aligned}
T\left(x_{i}\right) & =H_{\beta}\left(x_{i}\right) \theta_{\beta}=H_{\beta}\left(x_{i}\right) D_{\beta m} b^{m} ; \\
i & =1,2 ; \alpha=1,2, \ldots, N, m=0, \ldots, n-1 ;
\end{aligned}
$$

Therefore, the temperature gradients are similarly determined as

$$
\begin{aligned}
T_{, j} & =H_{\beta, j} \theta_{\beta}=H_{\beta, j} D_{\beta m} b^{m}, \\
i & =1,2, m=0, \ldots, n-1 .
\end{aligned}
$$

Further we notice that the input random quantity in above equations becomes the design input parameter $(h=b)$. The key feature of the proposed approach is to determine numerically the coefficients $D_{\beta m}$ for each node of the initial FEM mesh and each power of the polynomial representations of the nodal temperatures. This representation is developed by a multiple solution of the boundary value (and then also transient) problem around the mean value of the design parameter and then, using various approximation techniques, to recover the response function. To do so, the following extra algebraic systems of equations are formed and solved

$$
\left\{\begin{array}{c}
D_{\beta 1} b_{1}^{n-1}+D_{\beta 2} b_{1}^{n-2}+\ldots+D_{\beta n} b_{1}^{0}=\theta_{\beta}^{(1)} \\
D_{\beta 1} b_{2}^{n-1}+D_{\beta 2} b_{2}^{n-2}+\ldots+D_{\beta n} b_{2}^{0}=\theta_{\beta}^{(2)} \\
\ldots \\
D_{\beta 1} b_{n}^{n-1}+D_{\beta 2} b_{n}^{n-2}+\ldots+D_{\beta n} b_{n}^{0}=\theta_{\beta}^{(n)}
\end{array}\right.
$$

where the R.H.S. (right hand side) $\theta_{\beta}^{(j)}$ stands for the temperature in node $\beta=1, \ldots, N$ computed at $j$ th iteration step of the procedure consisting of sequential solution of the initial problem with incrementally modified value of the input parameter. The incrementing procedure is performed throughout the interval $[h-\Delta h, h+\Delta h]$ divided into $n$ equidistant sub-domains constituting the discrete values $h_{k}$. This formulation may be used in a local form also where the polynomial representation varies from node-to-node in the FEM mesh or may have a global character- to be found only once in some representative location. Effectively, we solve numerically the following algebraic system of equations with non-symmetric coefficients matrix $N$ times (it needs a different solver than that usually embedded into the FEM procedure for symmetric conductivity and capacity matrices):

$$
\left[\begin{array}{cccc}
\mathrm{h}_{1}^{\mathrm{n}-1} & \mathrm{~h}_{1}^{\mathrm{n}-2} & \ldots & \mathrm{h}_{1}^{0} \\
\mathrm{~h}_{2}^{\mathrm{n}-1} & \mathrm{~h}_{2}^{\mathrm{n}-2} & & \mathrm{~h}_{2}^{0} \\
\ldots & \ldots & & \ldots \\
\mathrm{h}_{\mathrm{n}}^{\mathrm{n}-1} & \mathrm{~h}_{\mathrm{n}}^{\mathrm{n}-2} & \ldots & \mathrm{h}_{\mathrm{n}}^{0}
\end{array}\right]\left\{\begin{array}{c}
\mathrm{D}_{\beta 1} \\
\mathrm{D}_{\beta 2} \\
\ldots \\
\mathrm{D}_{\beta n}
\end{array}\right\}=\left\{\begin{array}{c}
\theta_{\beta}^{(1)} \\
\theta_{\beta}^{(2)} \\
\ldots \\
\theta_{\beta}^{(n)}
\end{array}\right\}
$$

The unique solution for this system enables calculation of the necessary partial derivatives of the nodal responses $\theta_{\beta}(h)$ with respect to $h$ at the given $h_{0}$ as

$$
\begin{aligned}
\frac{\partial \theta_{\beta}}{\partial h}= & (n-1) D_{\beta n-1} h^{n-2}+(n-2) D_{\beta n-2} h^{n-3} \\
& +\ldots+D_{\beta 1},
\end{aligned}
$$

This differentiation has quite an analytical character and the increasing order partial derivatives of the nodal temperatures with respect to the design variable are not so much affected by the numerical errors from the increasing order equations solutions. It is also clear that the transient problem needs successive polynomial responses from time increment to the time increment, therefore for a discrete time moment $\tau$ (26) is reformulated in the following manner:

$\theta_{\beta}(\tau)=D_{\beta m}^{\tau} h^{m}, \quad m=0, \ldots, n-1 ; \beta=1, \ldots, N$.

Hence, it yields

$$
\begin{aligned}
T\left(x_{i}, \tau\right) & =H_{\beta}\left(x_{i}\right) \theta_{\beta}(\tau)=H_{\beta}\left(x_{i}\right) D_{\beta m}^{\tau} h^{m} ; \\
i & =1,2 ; \alpha=1,2, \ldots, N, m=0, \ldots, n-1 ;
\end{aligned}
$$

Therefore, the temperature gradients are similarly determined as

$$
\begin{aligned}
T_{, j}(\tau) & =H_{\beta, j} \theta_{\beta}(\tau)=H_{\beta, j} D_{\beta m}^{\tau} h^{m}, \\
i & =1,2, m=0, \ldots, n-1 .
\end{aligned}
$$

3.2 Finite Element equations for sensitivity gradients in elastoplasticity

Let us introduce a function of displacements increments $\Delta u_{k}(\mathbf{x})$ being a continuous and differentiable function over the region $\Omega$ consisting of the geometrically continuous subsets $\Omega_{e}$, where $e=1, \ldots, E$. It is assumed that the increments $\Delta u_{k}(\mathbf{x})$ are differentiable on the inter-element surfaces or the additional intervals $\partial \Omega_{e f}$ (for all $e, f=$ $1, \ldots, E, e \neq f$ ). Let us consider the following approximation of the displacements increments $\Delta u_{k}(\mathbf{x})$ for any $\mathbf{x} \in \Omega$ (Kowalczyk 2006):

$\Delta u_{k}(\mathbf{x})=\sum_{\zeta=1}^{N_{e}} \varphi_{\zeta k}(\mathbf{x}) \Delta q_{\zeta}^{(N)}$, 
where $\varphi_{\zeta k}(\mathbf{x})$ are the shape functions in the node $k, \Delta q_{\zeta}^{(N)}$ represent the nodal degrees of freedom vector, while $N_{\mathrm{e}}$ is the total number of those degrees of freedom in the considered node. Starting from the proposed approximation it is possible to express the gradients of the displacement vector as

$\Delta u_{k, l}(\mathbf{x})=\varphi_{k, l}^{\zeta}(\mathbf{x}) \Delta q_{\zeta}^{(N)}$.

Now, we introduce the following notation (Kleiber 1985)

$\bar{B}_{k l}^{(1) \zeta}(\mathbf{x})=\varphi_{k, l}^{\zeta}(\mathbf{x})$,

$\bar{B}_{k l}^{(2) \zeta}(\mathbf{x})=\varphi_{i, k}^{\zeta}(\mathbf{x}) \varphi_{i, l}^{\xi}(\mathbf{x}) q_{\xi}^{(N)}$,

$\overline{\bar{B}}_{k l}^{\zeta \xi}(\mathbf{x})=\frac{1}{2} \varphi_{i, k}^{\zeta}(\mathbf{x}) \varphi_{i, l}^{\xi}(\mathbf{x})$.

The strain tensor gradients are decomposed respectively into the linear and nonlinear parts as follows:

$\Delta \varepsilon_{k l}(\mathbf{x})=\Delta \bar{\varepsilon}_{k l}(\mathbf{x})+\Delta \overline{\bar{\varepsilon}}_{k l}(\mathbf{x})$

$\Delta \bar{\varepsilon}_{k l}(\mathbf{x})=\left[\bar{B}_{k l}^{(1) \zeta}+\bar{B}_{k l}^{(2) \zeta}\right] \Delta q_{\zeta}^{(N)}=\bar{B}_{k l}^{\zeta} \Delta q_{\zeta}^{(N)}$,

$\Delta \overline{\bar{\varepsilon}}_{k l}(\mathbf{x})=\overline{\bar{B}}_{k l}^{\zeta \xi} \Delta q_{\zeta}^{(N)} \Delta q_{\xi}^{(N)}$.

We return to the variational statement; there holds

$$
\begin{aligned}
& \frac{1}{2} C_{k l m n} \Delta \varepsilon_{k l} \Delta \varepsilon_{m n} \\
& =\frac{1}{2} C_{k l m n}\left(\Delta \bar{\varepsilon}_{k l}+\Delta \overline{\bar{\varepsilon}}_{k l}\right)\left(\Delta \bar{\varepsilon}_{m n}+\Delta \overline{\bar{\varepsilon}}_{m n}\right) \\
& =\frac{1}{2} C_{k l m n}\left(\Delta \bar{\varepsilon}_{k l} \Delta \bar{\varepsilon}_{m n}+\Delta \bar{\varepsilon}_{k l} \Delta \overline{\bar{\varepsilon}}_{m n}+\Delta \overline{\bar{\varepsilon}}_{k l} \Delta \bar{\varepsilon}_{m n}\right. \\
& \left.+\Delta \overline{\bar{\varepsilon}}_{k l} \Delta \overline{\bar{\varepsilon}}_{m n}\right) \\
& =\frac{1}{2} C_{k l m n}\left(\bar{B}_{k l}^{\zeta} \Delta q_{\zeta}^{(N)} \bar{B}_{m n}^{\xi} \Delta q_{\xi}^{(N)}\right. \\
& +\bar{B}_{k l}^{\zeta} \Delta q_{\zeta}^{(N)} \overline{\bar{B}}_{m n}^{\mu \nu} \Delta q_{\mu}^{(N)} \Delta q_{\nu}^{(N)} \\
& +\overline{\bar{B}}_{k l}^{\zeta \xi} \Delta u_{\zeta}^{(N)} \Delta q_{\xi}^{(N)} \bar{B}_{m n}^{\mu} \Delta q_{\mu}^{(N)} \\
& \left.+\overline{\bar{B}}_{k l}^{\zeta \xi} \Delta q_{\zeta}^{(N)} \Delta q_{\zeta}^{(N)} \overline{\bar{B}}_{m n}^{\mu \nu} \Delta q_{\mu}^{(N)} \Delta q_{\nu}^{(N)}\right) \\
& \frac{1}{2} \tilde{\sigma}_{k l} \Delta u_{i, k} \Delta u_{i, l}=\frac{1}{2} \tilde{\sigma}_{k l} \varphi_{i, k}^{\zeta}(\mathbf{x}) \Delta q_{\zeta}^{(N)} \varphi_{i, l}^{\xi}(\mathbf{x}) \Delta q_{\xi}^{(N)} .
\end{aligned}
$$

The following elemental stiffnesses are introduced in turn

$k_{\zeta \xi}^{(\sigma) e}=\int_{\Omega_{e}} \tilde{\sigma}_{k l} \varphi_{i, k}^{\zeta}(\mathbf{x}) \varphi_{i, l}^{\xi}(\mathbf{x}) d \Omega$,

$k_{\zeta \xi}^{(c o n) e}=\int_{\Omega_{e}} \frac{1}{2} C_{k l m n} \bar{B}_{k l}^{(1) \zeta} \bar{B}_{m n}^{(1) \xi} d \Omega$,

$$
\begin{aligned}
k_{\zeta \xi}^{(u) e}=\int_{\Omega_{e}} \frac{1}{2} C_{k l m n}( & \bar{B}_{k l}^{(1) \zeta} \bar{B}_{m n}^{(2) \xi}+\bar{B}_{k l}^{(2) \zeta} \bar{B}_{m n}^{(1) \xi} \\
& \left.+\bar{B}_{k l}^{(2) \zeta} \bar{B}_{m n}^{(2) \xi}\right) d \Omega,
\end{aligned}
$$

Where the entire elemental stiffness is a sum of those components

$k_{\zeta \xi}^{(1) e}=k_{\zeta \xi}^{(\sigma) e}+k_{\zeta \xi}^{(c o n) e}+k_{\zeta \xi}^{(u) e}$,

while the second and the third order stiffnesses are equal, respectively

$k_{\zeta \xi \mu}^{(2) e}=\int_{\Omega_{e}} \frac{3}{2} C_{k l m n}\left(\bar{B}_{k l}^{\zeta} \overline{\bar{B}}_{m n}^{\xi \mu}+\overline{\bar{B}}_{k l}^{\zeta \xi} \bar{B}_{m n}^{\mu}\right) d \Omega$,

$k_{\zeta \xi \mu \nu}^{(3) e}=\int_{\Omega_{e}} 2 C_{k l m n} \overline{\bar{B}}_{k l}^{\zeta \xi} \overline{\bar{B}}_{m n}^{\mu \nu} d \Omega$.

Introducing of $k_{\zeta \xi}^{(i)}$ for $i=1,2,3$ into the functional $J\left(\Delta u_{k}\right)$ and applying local to global coordinates system transformation one may obtain the following statement:

$\Delta u_{\zeta}^{(N)}=a_{\zeta \alpha} \Delta q_{\alpha}$.

Further, we obtain the energy functional in a discrete form as

$$
\begin{aligned}
J\left(\Delta q_{\alpha}\right)= & \frac{1}{2} K_{\alpha \beta}^{(1)} \Delta q_{\alpha} \Delta q_{\beta}+\frac{1}{3} K_{\alpha \beta \gamma}^{(2)} \Delta q_{\alpha} \Delta q_{\beta} \Delta q_{\gamma} \\
& +\frac{1}{4} K_{\alpha \beta \gamma \delta}^{(3)} \Delta q_{\alpha} \Delta q_{\beta} \Delta q_{\gamma} \Delta q_{\delta}-\Delta Q_{\alpha} \Delta q_{\alpha}
\end{aligned}
$$

The stationarity of the functional $J\left(\Delta q_{\alpha}\right)$ leads to the following matrix equation being a basis for the relevant computational implementation:

$$
\begin{aligned}
& K_{\alpha \beta}^{(1)} \Delta q_{\beta}+K_{\alpha \beta \gamma}^{(2)} \Delta q_{\beta} \Delta q_{\gamma}+K_{\alpha \beta \gamma \delta}^{(3)} \Delta q_{\beta} \Delta q_{\gamma} \Delta q_{\delta} \\
& \quad=\Delta Q_{\alpha}
\end{aligned}
$$


fulfilled for any configuration of the region $\Omega$. Therefore, its partial differentiation w.r.t. to the parameter $h$ yields

$$
\begin{aligned}
& K_{\alpha \beta}^{(1)} \frac{\partial\left(\Delta q_{\beta}\right)}{\partial h}+K_{\alpha \beta \gamma}^{(2)} \frac{\partial\left(\Delta q_{\beta}\right)}{\partial h} \Delta q_{\gamma}+K_{\alpha \beta \gamma}^{(2)} \Delta q_{\beta} \frac{\partial\left(\Delta q_{\gamma}\right)}{\partial h} \\
& +K_{\alpha \beta \gamma \delta}^{(3)} \frac{\partial\left(\Delta q_{\beta}\right)}{\partial h} \Delta q_{\gamma} \Delta q_{\delta}+K_{\alpha \beta \gamma \delta}^{(3)} \Delta q_{\beta} \frac{\partial\left(\Delta q_{\gamma}\right)}{\partial h} \Delta q_{\delta} \\
& +K_{\alpha \beta \gamma \delta}^{(3)} \Delta q_{\beta} \Delta q_{\gamma} \frac{\partial\left(\Delta q_{\delta}\right)}{\partial h}=\frac{\partial \Delta Q_{\alpha}}{\partial h}-\frac{\partial K_{\alpha \beta}^{(1)}}{\partial h} \Delta q_{\beta} \\
& -\frac{\partial K_{\alpha \beta \gamma}^{(2)}}{\partial h} \Delta q_{\beta} \Delta q_{\gamma}-\frac{\partial K_{\alpha \beta \gamma \delta}^{(3)}}{\partial k} \Delta q_{\beta} \Delta q_{\gamma} \Delta q_{\delta}
\end{aligned}
$$

It is clear from this statement that practically for the partial derivatives of the generalized displacements increment in (54) is solved in the same way as for the system (53) but the right hand side is slightly modified using the solutions of the zeroth order system corrected with the partial derivatives of the system matrices to the design parameter.

The same RFM technique is applied here as for the transient problem, so that instead of the nodal temperatures vector we discretize now

- the structural displacements increments as

$$
\begin{aligned}
\Delta u_{\zeta} & =a_{\zeta \beta} \Delta q_{\beta}=a_{\zeta \beta} D_{\beta}^{(p)} h^{p}, \\
\mathrm{p} & =0, \ldots, \mathrm{n}-1 ; \beta, \zeta=1, \ldots, \mathrm{N}
\end{aligned}
$$

- increments of the strain tensor components

$$
\begin{aligned}
\Delta \varepsilon_{k l} & =\Delta \bar{\varepsilon}_{k l}+\Delta \overline{\bar{\varepsilon}}_{k l}=\bar{B}_{k l}^{\zeta} \Delta u_{\zeta}+\overline{\bar{B}}_{k l}^{\zeta \xi} \Delta u_{\zeta} \Delta u_{\xi} \\
& =\bar{B}_{k l}^{\zeta} a_{\zeta \alpha} \Delta q_{\alpha}+\overline{\bar{B}}_{k l}^{\zeta \xi} a_{\zeta \alpha} \Delta q_{\alpha} a_{\xi \beta} \Delta q_{\beta} \\
& =\bar{B}_{k l}^{\zeta} a_{\zeta \alpha} D_{\alpha p} h^{p}+\overline{\bar{B}}_{k l}^{\zeta \xi} a_{\zeta \alpha} D_{\alpha p} h^{p} a_{\xi \beta} D_{\beta r} h^{r} \\
\mathrm{p}, \mathrm{r} & =0, \ldots, \mathrm{n}-1 ; \alpha, \beta=1, \ldots, \mathrm{N} \\
\mathrm{k}, 1 & =1,2,3
\end{aligned}
$$

- increments of the second Piola-Kirchhoff stress tensor components as

$$
\begin{aligned}
\Delta \tilde{\sigma}_{i j}= & C_{i j k l} \Delta \varepsilon_{k l}=C_{i j k l}\left(\Delta \bar{\varepsilon}_{k l}+\Delta \overline{\bar{\varepsilon}}_{k l}\right) \\
= & C_{i j k l}\left(\bar{B}_{k l}^{\zeta} \Delta u_{\zeta}+\overline{\bar{B}}_{k l}^{\zeta \xi} \Delta u_{\zeta} \Delta u_{\xi}\right) \\
= & C_{i j k l}\left(\bar{B}_{k l}^{\zeta} a_{\zeta \alpha} \Delta q_{\alpha}+\overline{\bar{B}}_{k l}^{\zeta \xi} a_{\zeta \alpha} \Delta q_{\alpha} a_{\xi \beta} \Delta q_{\beta}\right) \\
= & C_{i j k l}\left(\bar{B}_{k l}^{\zeta} a_{\zeta \alpha} D_{\alpha p} h^{p}\right. \\
& \left.\quad+\overline{\bar{B}}_{k l}^{\zeta \xi} a_{\zeta \alpha} D_{\alpha p} h^{p} a_{\xi \beta} D_{\beta r} h^{r}\right) \\
\mathrm{p}, \mathrm{r}= & 0, \ldots, \mathrm{n}-1 ; \alpha, \beta=1, \ldots, \mathrm{N} ; \\
\mathrm{i}, \mathrm{j}, \mathrm{k}, 1= & 1,2,3 ;
\end{aligned}
$$

Further, there holds

$$
\begin{aligned}
\frac{\partial \Delta u_{\zeta}}{\partial h} & =a_{\zeta \beta} \frac{\partial \Delta q_{\beta}}{\partial h}=a_{\zeta \beta} \frac{\partial\left(D_{\beta}^{(p)} b^{p}\right)}{\partial h}=p a_{\zeta \beta} D_{\beta}^{(p)} h^{p-1} \\
\mathrm{p} & =0, \ldots, \mathrm{n}-1 ; \beta=1, \ldots, \mathrm{N},
\end{aligned}
$$

Further, we determine the strain tensor components partial derivatives with respect to the input variable $h$ as

$$
\begin{aligned}
\frac{\partial \Delta \varepsilon_{k l}}{\partial h}= & \frac{\partial \Delta \bar{\varepsilon}_{k l}}{\partial h}+\frac{\partial \Delta \overline{\bar{\varepsilon}}_{k l}}{\partial h}=\bar{B}_{k l}^{\zeta} \frac{\partial \Delta u_{\zeta}}{\partial h}+\overline{\bar{B}}_{k l}^{\zeta \xi} \frac{\partial \Delta u_{\zeta}}{\partial h} \Delta u_{\xi} \\
& +\overline{\bar{B}}_{k l}^{\zeta \xi} \Delta u_{\zeta} \frac{\partial \Delta u_{\xi}}{\partial h}=\bar{B}_{k l}^{\zeta} a_{\zeta \alpha} \frac{\partial \Delta q_{\alpha}}{\partial h} \\
& +\overline{\bar{B}}_{k l}^{\zeta \xi} a_{\zeta \alpha} \frac{\partial \Delta q_{\alpha}}{\partial h} a_{\xi \beta} \Delta q_{\beta}+\overline{\bar{B}}_{k l}^{\zeta \xi} a_{\zeta \alpha} \Delta q_{\alpha} a_{\xi \beta} \frac{\partial \Delta q_{\beta}}{\partial h} \\
= & \bar{B}_{k l}^{\zeta} a_{\zeta \alpha} p D_{\alpha p} h^{p-1}+\overline{\bar{B}}_{k l}^{\zeta \xi} a_{\zeta \alpha} D_{\alpha p} p h^{p-1} a_{\xi \beta} D_{\beta r} h^{r} \\
& +\overline{\bar{B}}_{k l}^{\zeta \xi} a_{\zeta \alpha} D_{\alpha p} h^{p} a_{\xi \beta} D_{\beta r} r h^{r-1} \\
\mathrm{p}, \mathrm{r}= & 0, \ldots, \mathrm{n}-1 ; \beta=1, \ldots, \mathrm{N} ; \mathrm{k}, 1=1,2,3 ;
\end{aligned}
$$

Finally, the stress tensor components partial derivatives with respect to the input design variable

$$
\begin{aligned}
\frac{\partial \Delta \tilde{\sigma}_{i j}}{\partial h}= & \frac{\partial}{\partial h}\left(C_{i j k l} \Delta \varepsilon_{k l}\right) \\
= & \frac{\partial C_{i j k l}}{\partial h}\left(\Delta \bar{\varepsilon}_{k l}+\Delta \overline{\bar{\varepsilon}}_{k l}\right)+C_{i j k l}\left(\frac{\partial \Delta \bar{\varepsilon}_{k l}}{\partial h}+\frac{\partial \Delta \overline{\bar{\varepsilon}}_{k l}}{\partial h}\right) \\
= & \frac{\partial C_{i j k l}}{\partial h}\left(\bar{B}_{k l}^{\zeta} \Delta u_{\zeta}+\overline{\bar{B}}_{k l}^{\zeta \xi} \Delta u_{\zeta} \Delta u_{\xi \xi}\right) \\
& +C_{i j k l}\left(\bar{B}_{k l}^{\zeta} \frac{\partial \Delta u_{\zeta}}{\partial h}+\overline{\bar{B}}_{k l}^{\zeta \xi} \frac{\partial \Delta u_{\zeta}}{\partial h} \Delta u_{\xi}\right. \\
& \left.+\overline{\bar{B}}_{k l}^{\zeta \xi} \Delta u_{\zeta} \frac{\partial \Delta u_{\xi}}{\partial h}\right) \\
= & \frac{\partial C_{i j k l}}{\partial h}\left(\bar{B}_{k l}^{\zeta} a_{\zeta \alpha} \Delta q_{\alpha}+\overline{\bar{B}}_{k l}^{\zeta \xi} a_{\zeta \alpha} \Delta q_{\alpha} a_{\xi \beta} \Delta q_{\beta}\right) \\
& +C_{i j k l}\left(\bar{B}_{k l}^{\zeta} a_{\zeta \alpha} \frac{\partial \Delta q_{\alpha}}{\partial h}+\overline{\bar{B}}_{k l}^{\zeta \xi} a_{\zeta \alpha} \frac{\partial \Delta q_{\alpha}}{\partial h} a_{\xi \beta} \Delta q_{\beta}\right. \\
& \left.\quad+\overline{\bar{B}}_{k l}^{\zeta \xi} a_{\zeta \alpha} \Delta q_{\alpha} a_{\xi \beta} \frac{\partial \Delta q_{\beta}}{\partial h}\right) \\
= & \frac{\partial C_{i j k l}}{\partial h}\left(\bar{B}_{k l}^{\zeta} a_{\zeta \alpha} D_{\alpha p} h^{p}+\overline{\bar{B}}_{k l}^{\zeta \xi} a_{\zeta \alpha} D_{\alpha p} h^{p} a_{\xi \beta} D_{\beta r} h^{r}\right) \\
& +C_{i j k l}\left(\bar{B}_{k l}^{\zeta} a_{\zeta \alpha} D_{\alpha p} p h^{p-1}\right. \\
& +\overline{\bar{B}}_{k l}^{\zeta \xi} a_{\zeta \alpha} D_{\alpha p} p h^{p-1} a_{\xi \beta} D_{\beta r} h^{r} \\
& \left.+\overline{\bar{B}}_{k l}^{\zeta \xi} a_{\zeta \alpha} D_{\alpha p} h^{p} a_{\xi \beta} D_{\beta r} r h^{r-1}\right) \\
\mathrm{p}, \mathrm{r}, \ldots, \mathrm{n}- & ; \alpha, \beta=1, \ldots, \mathrm{N} ;
\end{aligned}
$$

$\mathrm{i}, \mathrm{j}, \mathrm{k}, \mathrm{l}=1,2,3$; 
Traditionally, to compare some sensitivities with respect to quite different design variables, the additional normalization procedure is finally carried out.

\section{Computational experiments}

4.1 Sensitivity gradients for the transient heat transfer in the homogeneous slab

This computational study is devoted to the heating of the homogeneous and isotropic rod with the constant crosssectional area and length $L=2.0$, the heat conductivity is taken as $\lambda=0.10$ and the heat capacity as $c=1.0$. The temperature is fixed at the left edge as $T=0$, the heat flux is applied at the right corner, whereas the entire structure is divided into 103 -noded parabolic finite elements and the time increment equals to $\Delta t=2 \mathrm{~s}$ (with their total number $\mathrm{n}=50$ ). Larger part of the computational experiment is conducted in the symbolic platform of MAPLE, v. 13, where the local response function for the additional time increments are determined and the sensitivity gradients are computed; the basic deterministic solutions to this problem have been provided externally by the academic FEM code. The 11 point discretization is used to define the numerical probing process around the mean values of the design variables of heat conductivity and also the capacity (basic increments are $\Delta h=0.10 h$ of those parameters mean values); therefore, an interval $[\mathrm{h}-5 \Delta h, h+5 \Delta h]$ was used to prepare the polynomial approximation for further differentiation.

The main results of the analysis are presented in Figs. 1, 2, 3, 4 and 5, where the horizontal axis presents each time the spatial domain of the rod, while the vertical one-the temperatures (Fig. 1), the first order temperature gradients with respect to heat capacity (Fig. 2), heat conductivity (Fig. 3) and their second gradients (Figs. 4 and 5), respectively. They are all presented in addition to some specific time increments; the agreement of the temperature distribution with that provided in Carslaw and Jeager (1959) is very good. As it is shown in Fig. 1, the total number of increments combined with input $\Delta \mathrm{t}$ resulted in almost steady-state conditions for the last increment.

First order sensitivities of the temperature field with respect to the heat capacity significantly change in time. Initially, the gradients distribution is positive everywhere and smoothly increases from 0 [for the boundary with $\mathrm{T}=0$ ] to the heated edge. Then, for $t=10 \mathrm{~s}$ and $\mathrm{t}=20 \mathrm{~s}$ those gradients increase almost everywhere remaining nonnegative and resulting in continuous as well as smooth distributions; the only exception is noticed at the left corner where some negative values appear. Then, the sensitivities of the temperature start to systematically decrease along the heated rod,

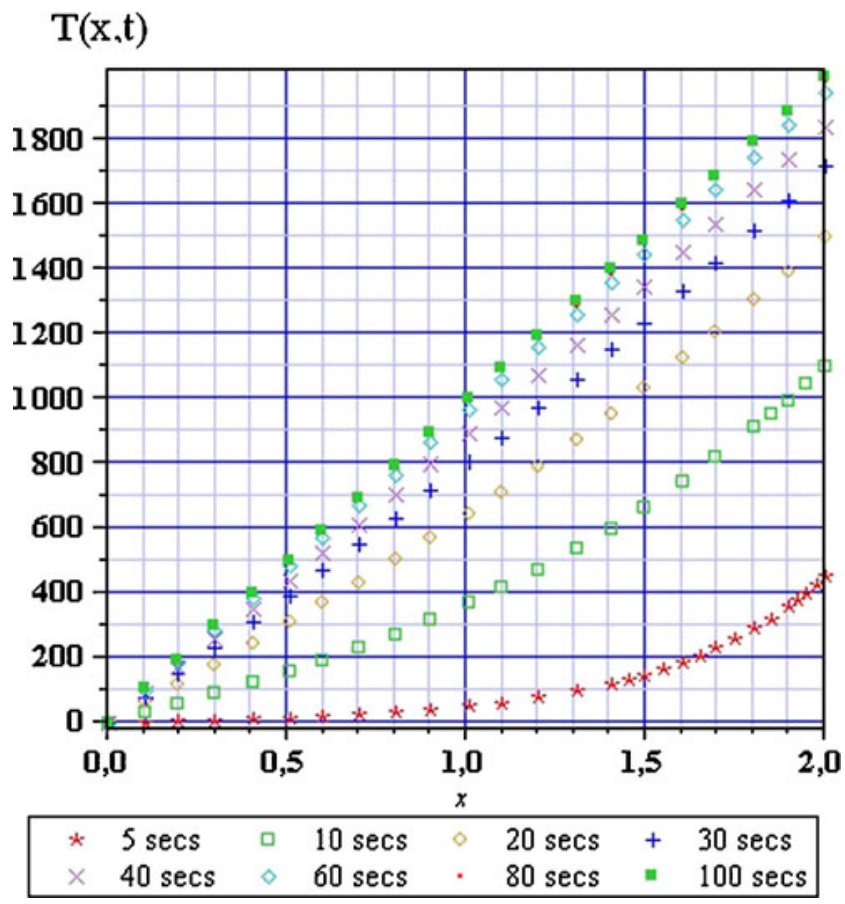

Fig. 1 Temperature history approximated by the RFM-FEM

however some local variations at $\mathrm{x}=0$ are still noticed. All those tendencies reflect intuitively clear tendency that the heat capacity is as long influential as the heat wave penetrates the rod. Once heat flow stabilizes and tends slowly to the steady state, the heat capacity of the system appears to be almost negligible.

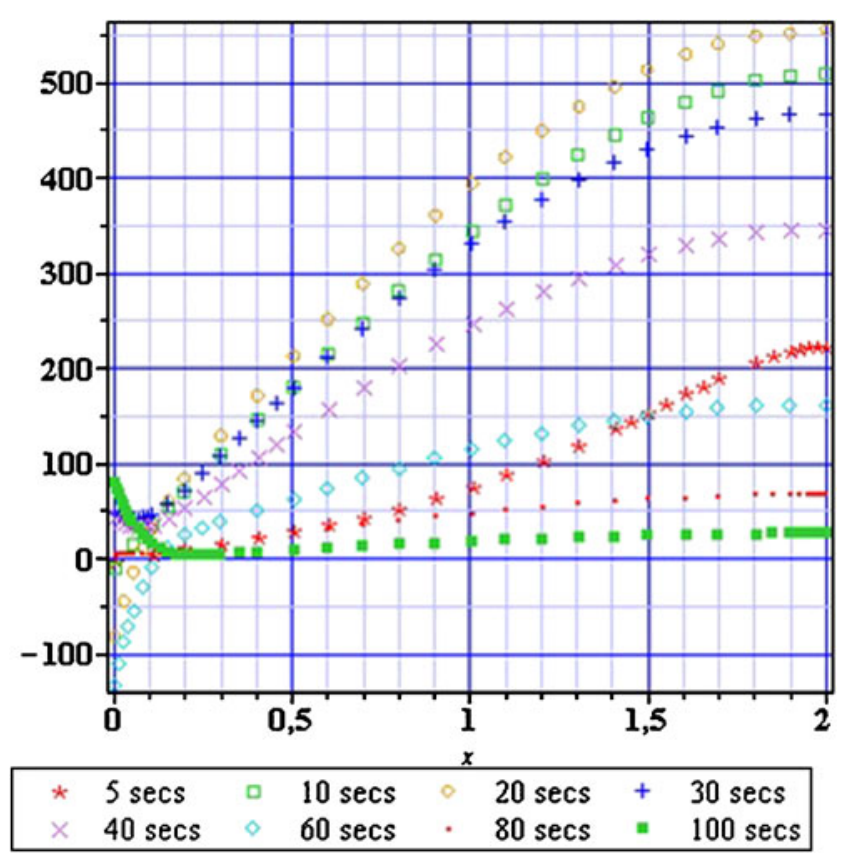

Fig. 2 First order temperature sensitivity gradients to heat capacity 


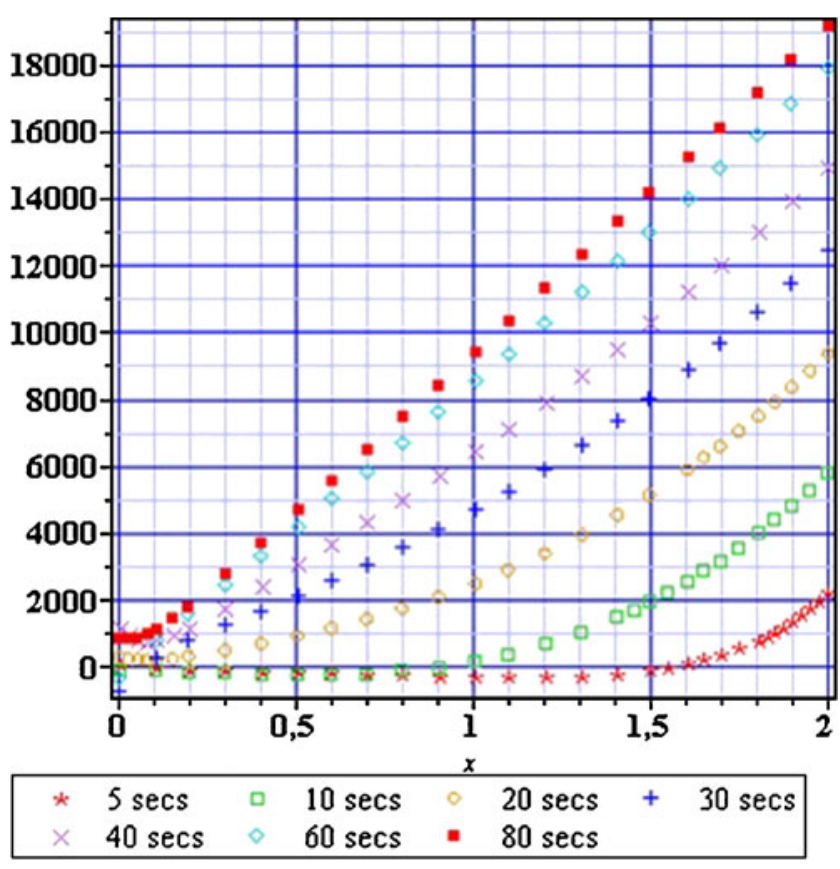

Fig. 3 First order temperature sensitivity gradients to heat conductivity

The temperature gradients with respect to heat conductivity (see Fig. 3) seem to have the spatial distributions similar to the temperature variations given in Fig. 1. Those gradients increase from the left corner of the rod to the heated end-through some small negative numbers at the process beginning to the apparently positive values at the end,

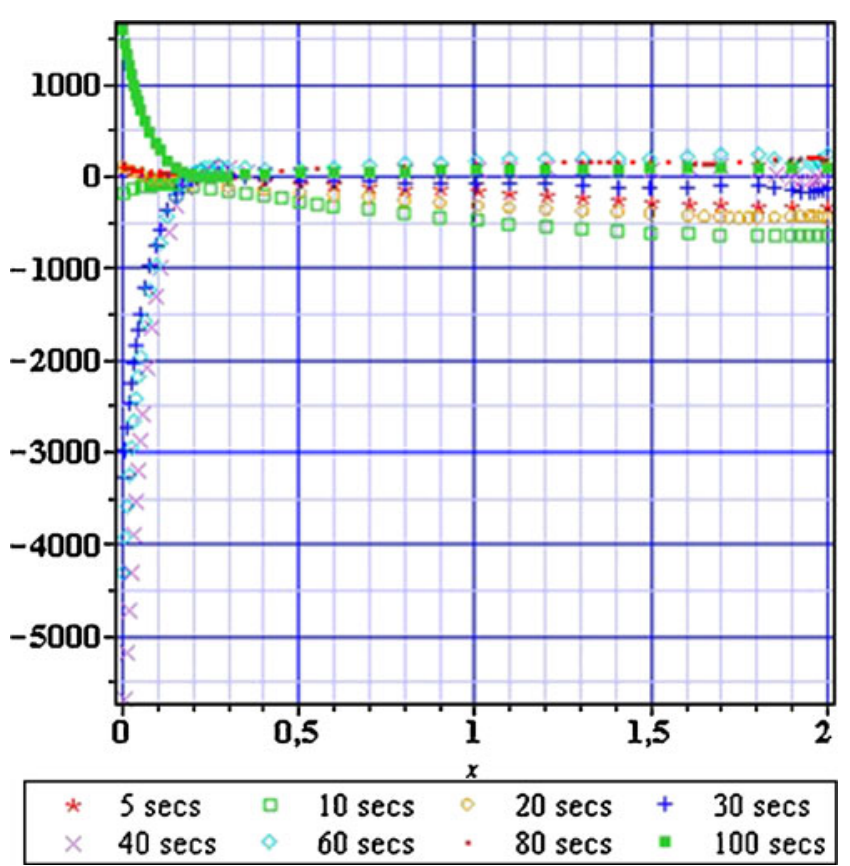

Fig. 4 Second order temperature sensitivity gradients to heat capacity

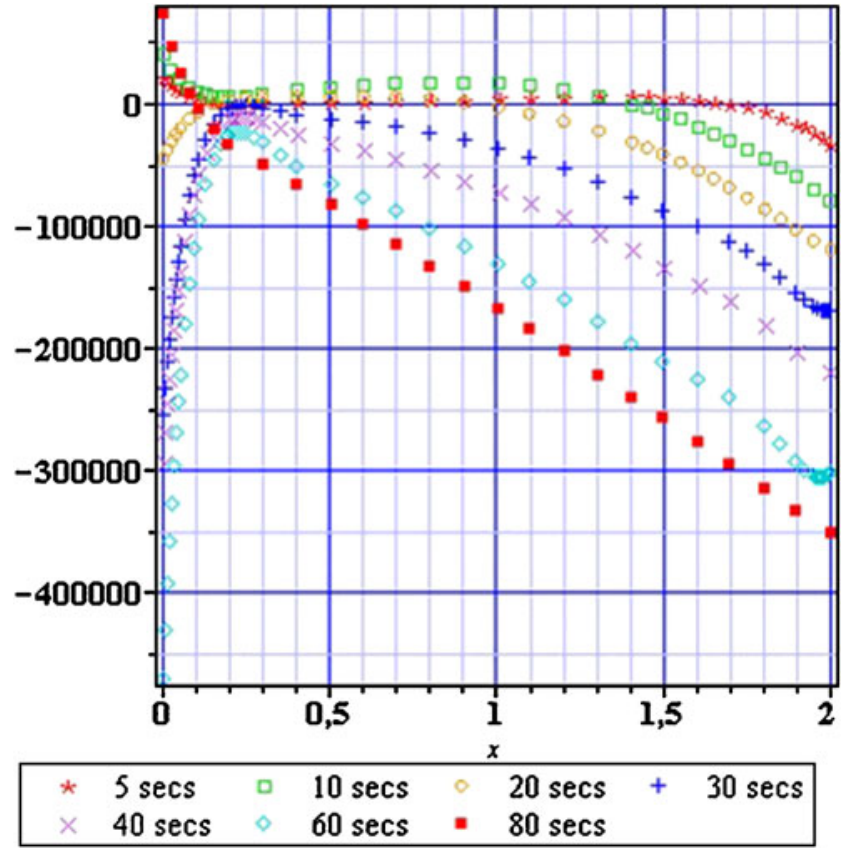

Fig. 5 Second order temperature sensitivity gradients to heat conductivity

forming almost straight line for $t=80 \mathrm{~s}$. Those gradients stabilize like the temperatures together with time - the differences between neighbouring curves systematically decrease.

Next two figures, Figs. 4 and 5, presenting the second order sensitivities with respect to heat capacity and conductivity enable to analyze the convexity of the additional temperature variations. Both gradients time fluctuations exhibit similar largest oscillations close to $\mathrm{x}=0$ with both positive and negative values but they have completely different character on the remaining part of this rod (almost 0 in Fig. 4 and with descending tendency in Fig. 5). The gradients with respect to heat capacity oscillate around 0 value for $t=5 \mathrm{~s}$, then negative values dominate with the steepest descent for $\mathrm{x}$ tending to 0 . They tend to positive values for all $\mathrm{x}$ belonging to the interval $[0, \mathrm{~L}]$ with absolute maximum at $\mathrm{x}=0$, so that concave distribution of the temperature close to $t=0$ become effectively convex at the end of the heating process. The gradients in Fig. 5 starting from almost 0 values evolve through small positive values (at $10 \mathrm{~s}$ ) to change to entirely negative distribution having maximum close to $\mathrm{x}=0.2$ and minimum for $\mathrm{x}$ tending to 0 . Finally, for $x$ close to 0 , those gradients appear to be positive for $0.0<\mathrm{x}<0.10$ nonlinearly decreasing within this interval to 0 and then almost linearly decrease to negative minimum at the right corner of the structure. So that the spatial temperature variations with respect to heat conductivity remain concave almost always and everywhere in the heated rod. 
Table 1 CFD versus RFM first order temperature gradients

\begin{tabular}{lcccc}
\hline$t[\mathrm{~s}]$ & $\frac{\partial T(x=1.0, t)}{\partial c}$ RFM & $\frac{\partial T(x=1.0, t)}{\partial c}$ FDM & $\frac{\partial T(x=1.0, t)}{\partial \lambda}$ RFM & $\frac{\partial T(x=1.0, t)}{\partial \lambda}$ FDM \\
\hline 5 & 75,199 & 76,015 & $-279,482$ & $-280,500$ \\
10 & 343,662 & 345,200 & 186,361 & 176,500 \\
20 & 392,920 & 393,150 & $2.489,539$ & $2.480,500$ \\
30 & 329,751 & 329,050 & $4.699,984$ & $4.700,000$ \\
40 & 245,775 & 244,850 & $6.423,351$ & $6.436,000$ \\
60 & 115,132 & 114,700 & $8.497,903$ & $8.540,500$ \\
80 & 47,950 & 47,959 & $9.407,844$ & $9.476,000$ \\
100 & 18,767 & 18,900 & $9.777,594$ & $9.860,500$ \\
\hline
\end{tabular}

The results of the RFM analysis are finally contrasted with the finite difference scheme based on the central difference rule. The results are contained in Table 1 and they obey the first partial derivatives of the temperature at the midpoint of the heated structure for the few time moments specified in the first column of this table. The finite difference computations use as the perturbation parameter the basic interval from the RFM approach and the direct neighbouring values to the mean value of the design parameter. One may conclude here that the differences between those two methods are negligible (only accidentally larger than $1 \%$ ). This perfect agreement may result from the fact that the structure is homogeneous and the chosen parameters are constant into it.

\subsection{Elastoplastic plane truss sensitivity computations}

A demonstration of the RFM-FEM to the design sensitivity analysis in the nonlinear structural problem is carried out on the example of the small deformation of the elastoplastic plane truss structure presented in Fig. 6; Young modulus has mean value of $30 \mathrm{GPa}$ and is treated as the design parameter of this study. The entire computational procedure

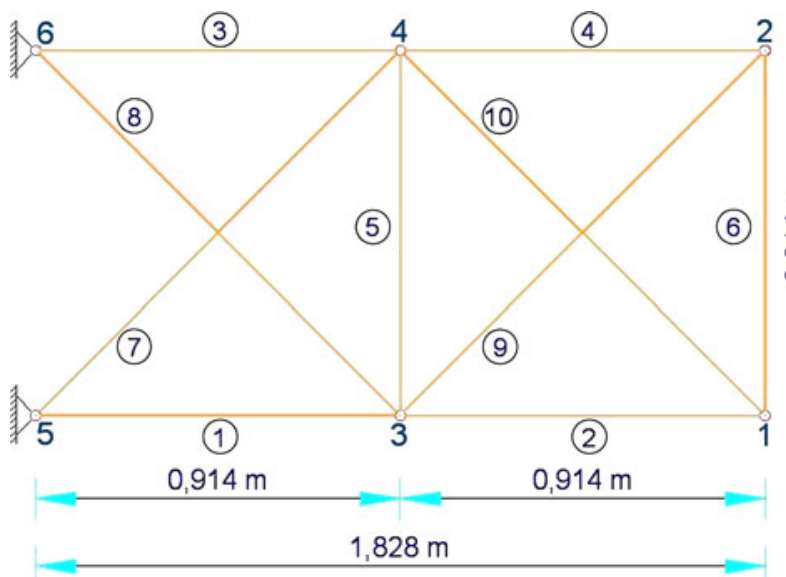

Fig. 6 The plane truss structure is performed using the classical academic FEM software (elasto-plastic analysis, 2D linear truss finite elements) and the symbolic computational algebra system MAPLE. Using this hybrid computational package we examine the maximum vertical displacements in the node 1 , where the vertical loading acting downwards is applied. It was impossible to make a presentation for all nodes (or degrees of freedom) since the structure has discrete and multidimensional character unlike in the previous case.

The set of all input data enabled for the nine increments in the analysis, so that each time the response function of this displacement with respect to the Young modulus of the structure was recovered using nine-point approximation consistent with the above considerations (see Fig. 7). All the polynomial interpolations given in this figure show that the approximants are continuous and smooth function of the input parameter; they exhibit monotonous behavior

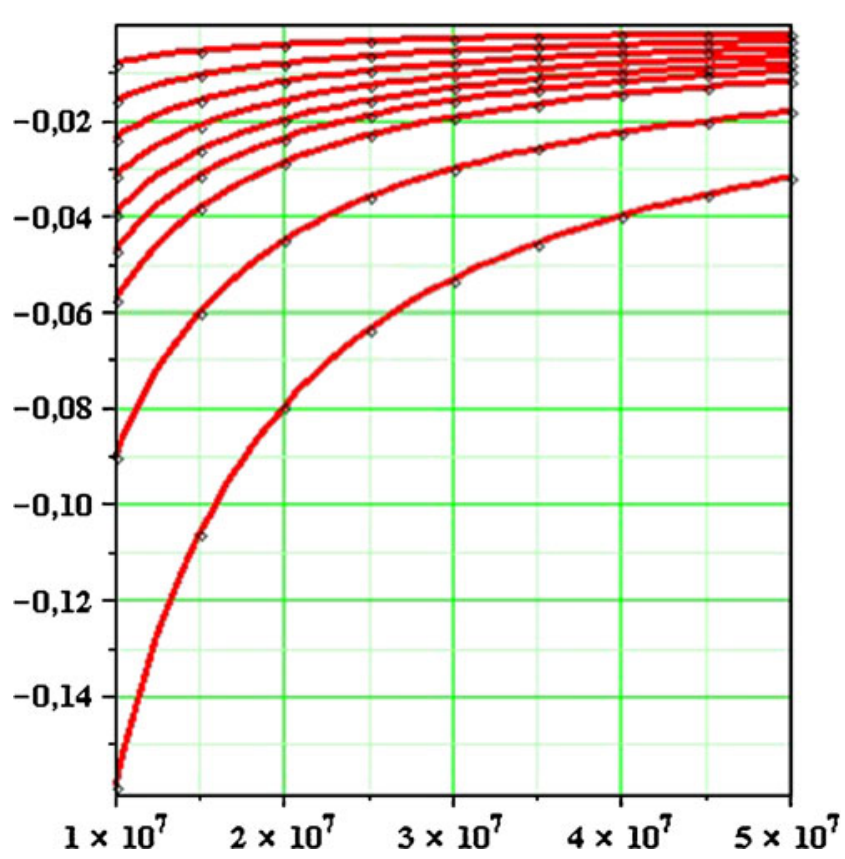

Fig. 7 The response functions for all increments of the vertical displacements, 1 st node 


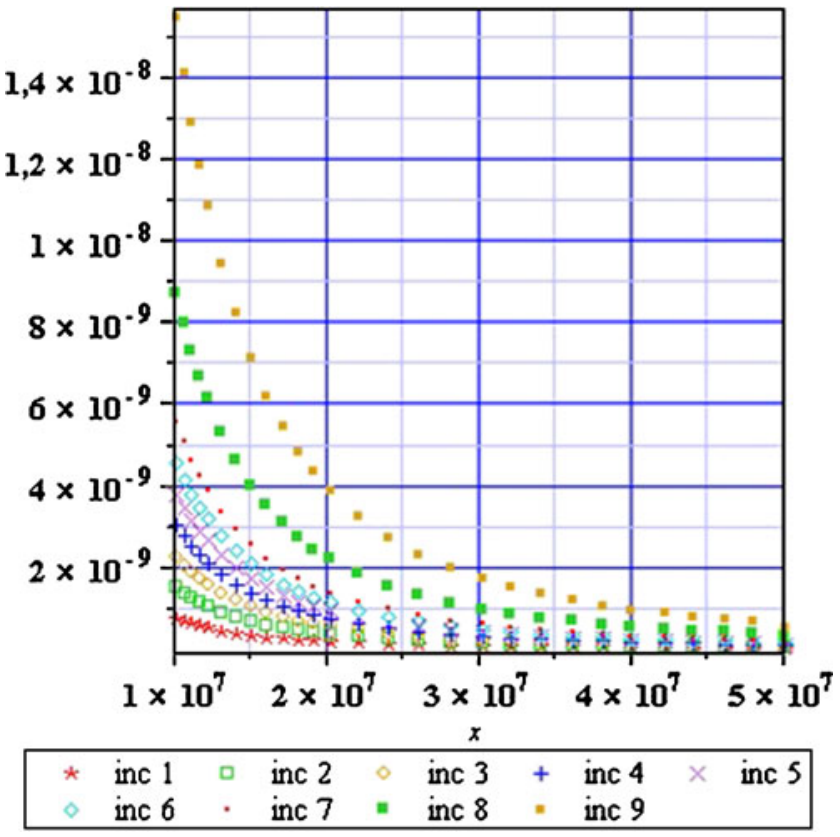

Fig. 8 The first order sensitivities of the vertical displacement, 1st node

for all increments (noticed on the graph). As one may expect, the larger Young modulus (marked on the horizontal axis) the less vertical displacements computed and they systematically increase together with the increment number.

Figures 8 and 9 demonstrate the full effectiveness of the RFM-FEM approach, where the first and the second order

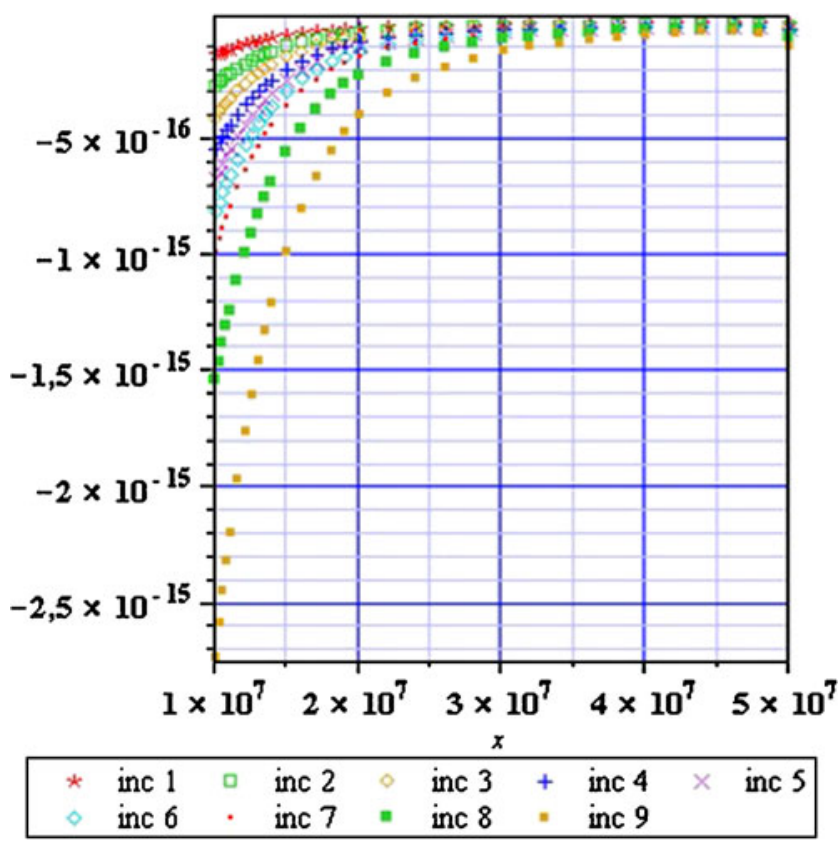

Fig. 9 The second order sensitivities of the vertical displacement, 1st node
Table 2 CFD versus RFM first order vertical displacement increments sensitivities

\begin{tabular}{lll}
\hline Increment number & $\frac{\partial q_{1}}{\partial E}$ RFM & $\frac{\partial q_{1}}{\partial E}$ FDM \\
\hline 1 & $8,692 \mathrm{E}-11$ & $8,949 \mathrm{E}-11$ \\
2 & $1,739 \mathrm{E}-10$ & $1,789 \mathrm{E}-10$ \\
3 & $2,608 \mathrm{E}-10$ & $2,684 \mathrm{E}-10$ \\
4 & $3,477 \mathrm{E}-10$ & $3,579 \mathrm{E}-10$ \\
5 & $4,345 \mathrm{E}-10$ & $4,474 \mathrm{E}-10$ \\
6 & $5,214 \mathrm{E}-10$ & $5,369 \mathrm{E}-10$ \\
7 & $6,362 \mathrm{E}-10$ & $6,550 \mathrm{E}-10$ \\
8 & $9,978 \mathrm{E}-10$ & $1,027 \mathrm{E}-9$ \\
9 & $1,766 \mathrm{E}-9$ & $1,818 \mathrm{E}-9$ \\
\hline
\end{tabular}

derivatives are numerically determined not only for the main value of the design parameter $E$ but also for the entire computational domain of the response function. If one needs to recover the particular values for the given Young modulus the intersection points of the vertical line with this family of curves return the desired results. Both the local response and its first two derivatives are continuous and monotonous functions without any local singularities nor numerical instabilities. As it is seen in Fig. 8, the smaller value of the design parameter, the larger values of the first derivative for the entire incrementation procedure. Figure 9 apparently shows that the response functions for the displacement being studied are convex nowhere, which perfectly coincides with Fig. 7. Let us finally note that theoretically any order partial derivatives of the state parameters w.r.t. some input quantities may be found providing that sufficiently dense representation for the RFM is done. This aspect is of the special importance considering further implementations of the generalized perturbation-based Stochastic Finite Element Method (Kamiński 2009a).

The particular numerical values of the vertical displacements in node 1 are collected in Table 2 for the RFM approach invented here and the classical CFD approach. The finite difference calculus is based here on the central difference formula employing the values surrounding the design parameter mean value. The RFM technique results in an underestimation of the FDM gradients for all increments of the elastoplastic analysis. The numerical error equals to about $1.5 \%$ of the exact partial derivative.

\subsection{Eigenvalue analysis of the high telecommunication tower}

The last computational experiment is devoted to determination of the eigenvibrations for the steel telecommunication tower with the height equal to $52.0 \mathrm{~m}$, the FEM discretiza- 


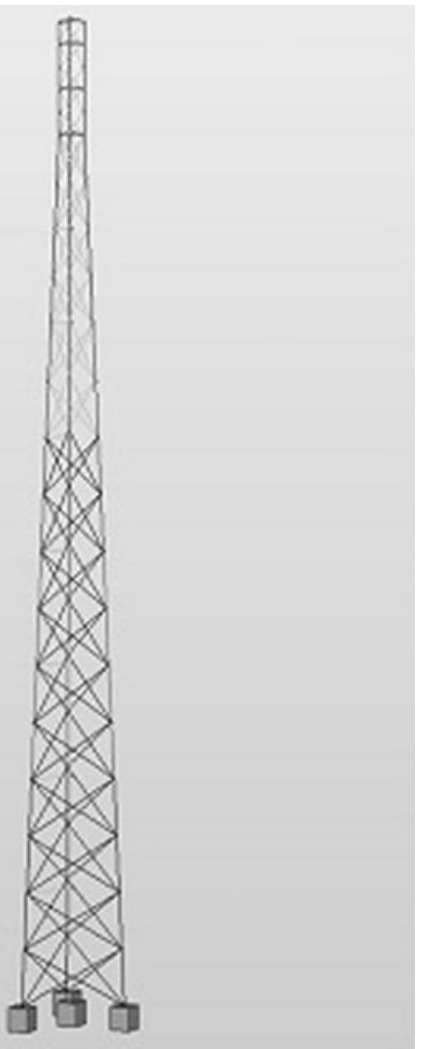

Fig. 10 FEM discretization of the telecommunication tower

tion of which has been schematically presented in Fig. 10. The entire structure has been discretized using the twonoded 183 linear space structure finite elements (3D truss elements) joined in 66 nodal points and clamped at the ground level. All the structural members have been manufactured with the stainless steel having Young modulus equal to $\mathrm{E}=205 \mathrm{GPa}$ treated here as design input variable. The results of an analysis have been presented in Figs. 11, 12 and 13, where we have in turn - the response functions of the particular eigenvalues with respect to Young modulus as well as the first and the second order partial derivatives of those eigenvalues. Since the eigenvalues have a global character for the engineering structures, also global polynomial approximation w.r.t. design parameter is necessary here. Hence, one may simply replace in (32) the nodal temperatures vector by the eigenvalues vector indexed with the natural numbers instead of the nodal points of the mesh and the remaining FEM discretization proceeds quite similarly.

As one may recognize from Fig. 11, some eigenvalues are extremely close to each other since they have transversal, torsional, longitudinal or complex character. Following this result, the first and the second order gradients are also grouped into three essentially different distributions.

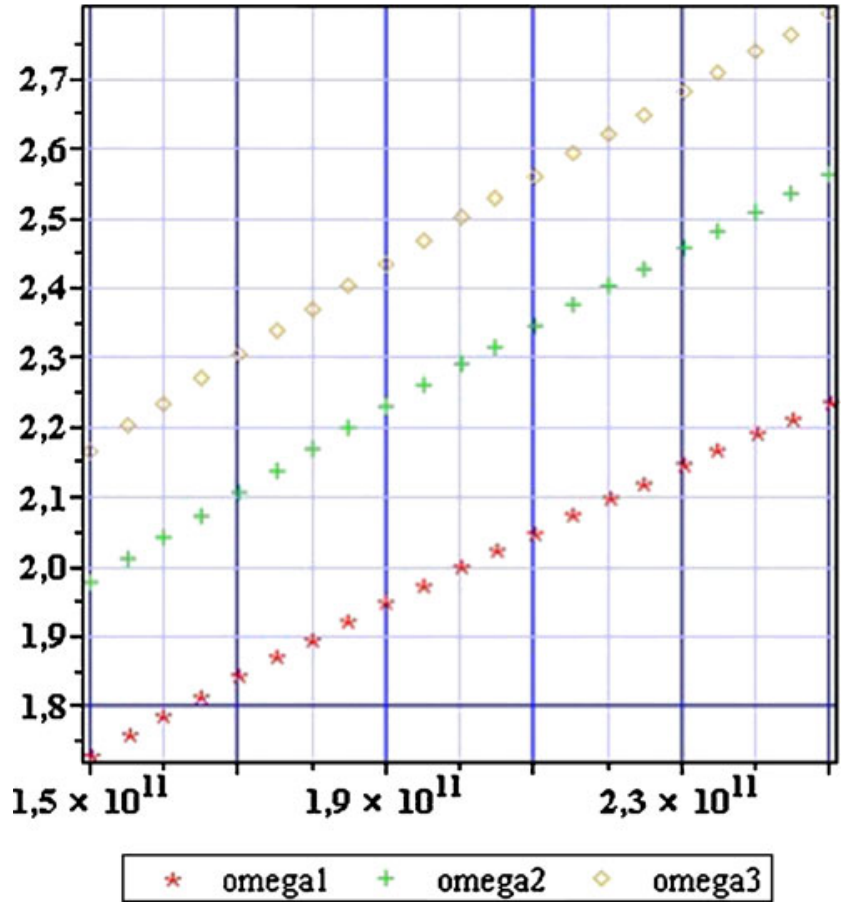

Fig. 11 Lower eigenvalues variations of the telecommunication tower

Generally, the first order derivatives are positive and the second derivatives - all negative, so that increasing of this structure Young modulus (its overall stiffness) leads to the

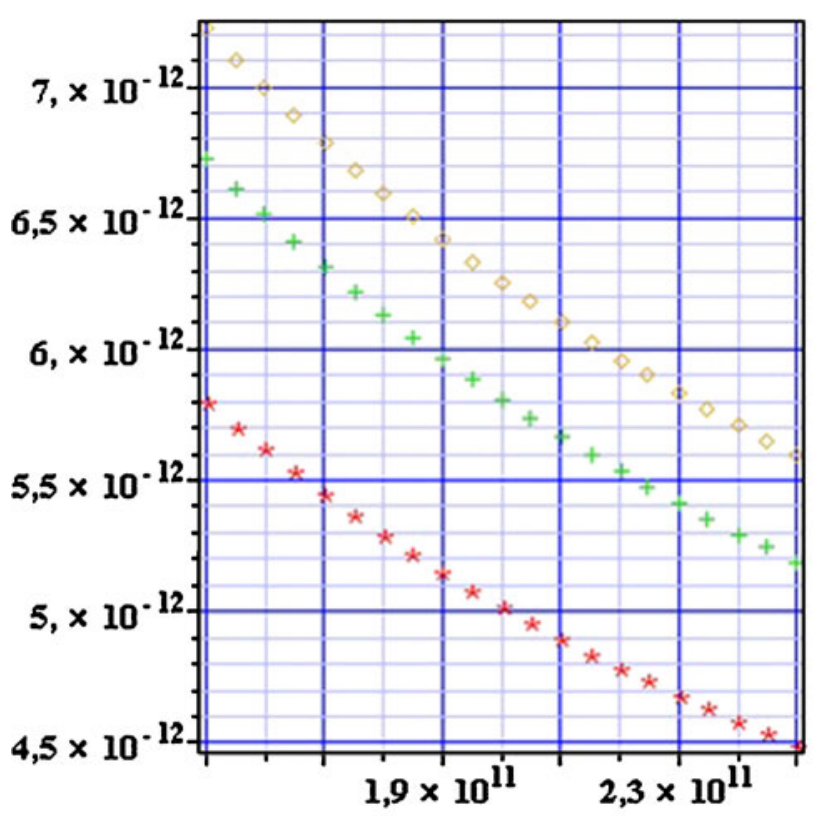

* domegal/dE + domega2/dE $\diamond$ domega3/dE

Fig. 12 First order partial derivatives of the lower eigenvalues w.r.t. Young modulus 


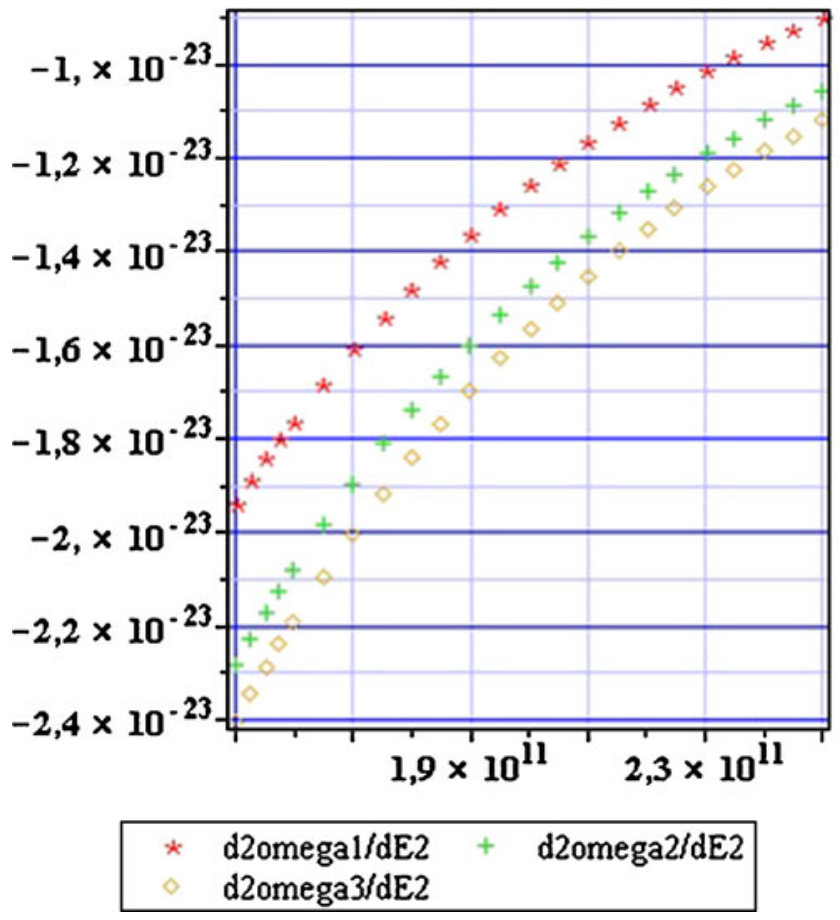

Fig. 13 Second order partial derivatives of the lower eigenvalues w.r.t. Young modulus

additional increase of all eigenvalues. The negative second order gradients reflect the fact that the higher stiffness, the less influence of Young modulus on the eigenvalues being computed. The computational time, thanks to the RFM application, approximately equals here to the single solution time (for the given number of eigenvalues) multiplied by the total number of polynomial approximation points (plus small additional time for the graphical post-processing procedures). This time is essentially larger than in the DDMbased perturbation technique implementation, however it guarantees automatic recovery of the higher order sensitivities at the same time, whereas the DDM technique needs an extra formation and the solution to the higher order equations also (it significantly enlarges the total time of computations).

Finally we compare the detailed numerical values of first and second sensitivity gradients for the studied eigenvalues determined using the Response Function Method and, independently, via the Central Finite Difference (CFD) approach. The corresponding data are collected in Table 3-the first three eigenvalues computed for the design parameter mean value as well as its lower and upper bounds within the variability interval. The first gradients obtained using the RFM approach for the mean value are underestimated in a comparison to the corresponding values computed using the CFD - the differences are in this case negligible because they are smaller than $1 \%$. Those differences are apparently larger at the end of design parameter variability interval. The RFM approach overestimates the finite differencing approach results at the lower bound (forward difference) and underestimates those results for the upper bound (backward difference). Computational error is significant here so that the partial derivatives may be reliably computed using the RFM approximation for the mean value of the design parameter only (or in its very close neighbourhood). Very similar situation takes place in the case of the second order gradients - the best agreement between the RFM and CFD techniques is obtained at the mean value of the design parameter. At the lower and upper bounds the RFM technique once more returns larger values than the CFD approach. Analyzing this comparison one needs to take into account the fact that the difference formulas include the lowest orders only, so that the real value of the particular derivatives may have some intermediate values in-between the results computed using both techniques.

\section{Concluding remarks}

The sensitivity analysis method based on the explicit determination of the local response functions relating the struc-
Table 3 CFD versus RFM first and second order eigenvalues sensitivities

\begin{tabular}{llllll}
\hline$\omega$ & $\omega_{i}$ & $\frac{\partial \omega_{i}}{\partial E}$ RFM & $\frac{\partial \omega_{i}}{\partial E}$ FDM & $\frac{\partial^{2} \omega_{i}}{\partial E^{2}}$ RFM & $\frac{\partial^{2} \omega_{i}}{\partial E^{2}}$ FDM \\
\hline $209 \mathrm{E} 9$ & $\omega_{1}$ & $4,954 \mathrm{E}-12$ & $4,980 \mathrm{E}-12$ & $-1,2124 \mathrm{E}-23$ & $-1,2284 \mathrm{E}-23$ \\
& $\omega_{2}$ & $5,736 \mathrm{E}-12$ & $5,766 \mathrm{E}-12$ & $-1,4134 \mathrm{E}-23$ & $-1,4355 \mathrm{E}-23$ \\
& $\omega_{3}$ & $6,176 \mathrm{E}-12$ & $6,208 \mathrm{E}-12$ & $-1,5080 \mathrm{E}-23$ & $-1,5277 \mathrm{E}-23$ \\
$41 \mathrm{E} 9$ & $\omega_{1}$ & $1,102 \mathrm{E}-11$ & $0,922 \mathrm{E}-12$ & $-1,1614 \mathrm{E}-22$ & $-1,0585 \mathrm{E}-22$ \\
& $\omega_{2}$ & $1,306 \mathrm{E}-11$ & $1,084 \mathrm{E}-11$ & $-1,4131 \mathrm{E}-22$ & $-1,2779 \mathrm{E}-22$ \\
& $\omega_{3}$ & $1,373 \mathrm{E}-11$ & $1,148 \mathrm{E}-11$ & $-1,4505 \mathrm{E}-22$ & $-1,3143 \mathrm{E}-22$ \\
$369 \mathrm{E} 9$ & $\omega_{1}$ & $3,669 \mathrm{E}-12$ & $3,798 \mathrm{E}-12$ & $-7.6558 \mathrm{E}-24$ & $-6,0083 \mathrm{E}-24$ \\
& $\omega_{2}$ & $4,231 \mathrm{E}-12$ & $4,383 \mathrm{E}-12$ & $-7,6440 \mathrm{E}-24$ & $-6,8650 \mathrm{E}-24$ \\
& $\omega_{3}$ & $4,569 \mathrm{E}-12$ & $4,735 \mathrm{E}-12$ & $-9,9355 \mathrm{E}-24$ & $-7,5550 \mathrm{E}-24$ \\
\hline
\end{tabular}


tural behaviour with the design input variables has been presented in this work. Since those response functions are numerically derived in the analytical, polynomial form, one can relatively easy (and with no additional time cost and computer power increase) determine also the additional higher order partial derivatives. Because the numerical implementation does not need any access to the original FEM code, it may be especially attractive for the large commercial systems users with complex material models (also in the unsteady conditions or material nonlinearities). The computational experiments proved that the hybrid FEMsymbolic implementation is especially efficient because of the usage of automatic any order analytical differentiation together with the very powerful graphical presentation options. Those higher order derivatives determination (as well as mixed partial differentiation also) leads to further possible application of this technique in the generalized perturbation-based Stochastic Finite Element Method. The RFM-FEM seems to be also applicable for the shape sensitivity problems (Dems and Mróz 1993; Laporte and Le Tallec 2003) as well as to stochastic design sensitivity analysis. A comparison of this new approach against the classical finite differencing scheme shows practically negligible differences between those two methods in all the FEM models - for transient, elastoplastic as well as for the eigenvibrations determination. Finally, it should be mentioned that the numerical verification of computational accuracy against the CFDM approach has been presented before in Kamiński (2009b) for the homogenization problem of the periodic (although linear elastic) fiber-reinforced composites, where elastic parameters of the components were taken as the design variables. Of course, the RFM approach may be efficiently used together with the other numerical methods (Collatz 1966; Kamiński 2010) (like Finite Difference or Boundary Element Method) in computational structural sensitivity analysis.
Acknowledgement This paper has been financially supported by the Polish Ministry of Science and Higher Education under the grant no 519386636.

Open Access This article is distributed under the terms of the Creative Commons Attribution Noncommercial License which permits any noncommercial use, distribution, and reproduction in any medium, provided the original author(s) and source are credited.

\section{References}

Bathe KJ (1996) Finite element procedures. Prentice Hall, Englewood Cliffs

Carslaw HS, Jeager JC (1959) Conduction of heat in solids. Oxford Univ Press, London

Collatz L (1966) The numerical treatment of differential equations, 3rd edn. Springer, New York

Dems K, Mróz Z (1993) On shape sensitivity approaches in the numerical analysis of structures. Struct Optim 6:86-92

Kamiński M (2009a) Perturbation-based stochastic finite element method using polynomial response function for the elastic beams. Mech Res Commun 36:381-390

Kamiński M (2009b) Sensitivity and randomness in homogenization of periodic fiber-reinforced composites via the response function method. Int J Solids Struct 46(3-4):923-937

Kamiński M (2010) Structural design sensitivity using boundary elements and polynomial response function. Struct Multidisc Optimiz 41:107-116

Kang BS, Park GJ, Arora JS (2006) A review of optimization of structures subjected to transient loads. Struct Multidisc Optim 31(2):81-95

Khuri AI, Cornell JA (1987) Response surfaces: design and analysis. Marcel Dekker, New York

Kleiber M (1985) Finite element method in nonlinear continuum mechanics (in Polish). Polish Sci Publ, Warszawa

Kleiber M et al (1997) Parameter sensitivity in nonlinear mechanics. Wiley, Chichester

Kowalczyk P (2006) Sensitivity analysis in finite element computations of elasto-plasticity. IFTR, Warsaw, PAS Report No 7

Laporte E, Le Tallec P (2003) Numerical methods in sensitivity analysis and shape optimisation. Birkhauser, Boston

Oden JT (1972) Finite elements of nonlinear continua. McGraw-Hill, New York 